Relations industrielles

Industrial Relations

\title{
Le The New Industrial State de John K. Galbraith
}

\section{Roger H. Stanton}

Volume 23, numéro 1, 1968

URI : https://id.erudit.org/iderudit/027870ar

DOI : https://doi.org/10.7202/027870ar

Aller au sommaire du numéro

Éditeur(s)

Département des relations industrielles de l'Université Laval

ISSN

0034-379X (imprimé)

1703-8138 (numérique)

Découvrir la revue

Citer cet article

Stanton, R. (1968). Le The New Industrial State de John K. Galbraith. Relations industrielles / Industrial Relations, 23(1), 159-164.

https://doi.org/10.7202/027870ar

Tous droits réservés (C Département des relations industrielles de l'Université Laval, 1968
Ce document est protégé par la loi sur le droit d'auteur. L'utilisation des services d’Érudit (y compris la reproduction) est assujettie à sa politique d'utilisation que vous pouvez consulter en ligne.

https://apropos.erudit.org/fr/usagers/politique-dutilisation/ 
(rente d'invalidité, rente de veuve ou de veuf invalide, rente d'orphelin), partiellement au " taux uniforme » $\$ 25$ par mois sujet à la majoration selon l'indice des rentes) et partiellement en fonction des gains admissibles, tout cela, nous le répétons, sans égard aux cotisations versées.

Vu que les taux de cotisations des régimes au Canada ne sont pas déterminés sur une base d'équivalence actuarielle et que les prestations ne sont pas exprimées en fonction des cotisations individuelles, il serait, du moins théoriquement possible, si on le voulait, de faire varier, de temps en temps, le taux de cotisations selon les cycles économiques; par exemple, réduire ce taux dans les périodes de récession ou de déflation et l'augmenter pendant les périodes de prospérité ou d'inflation. Une telle opinion personnelle a été exprimée par l'auteur de ces notes à plusieurs reprises aux Congrès Internationaux d'Actuaires (en 1951 en Hollande et en 1964 en Grande-Bretagne).

Il est cependant à remarquer que le procédé de faire varier le taux cie cotisation selon la situation économique du pays se heurte au Canada à un obstacle formel. Car aussi bien le R.R.Q. que le R.P.C. stipulent que toute loi modifiant les taux de contributions ne peut entrer en vigueur qu'à la date fixée par une proclamation du gouvernement respectif, mais que cette date ne doit pas être antérieure au premier jour de la troisième année suivant l'année au cours de laquelle le projet de la loi à ce sujet a été présenté au Parlement concerné.

Ainsi, si cette disposition (art. 222 du R.R.Q. et art. 115 du R.P.C.) n'est pas amendée, toute modification des taux de contributions pour tenir compte de la situation économique perdra sa raison d'être, car elle n'aura effet que plusieurs années après que la décision serait prise et pourra n'être plus nécessaire après une telle période.

\section{Le The New Industrial State de John K. Galbraith}

\section{Roger H. Stanton*}

The danger to liberty lies in the subordination of belief to the needs of the industrial system...

J.K.G.

Dans cet ouvrage ${ }^{1}$ John K. Galbraith, économiste de renom, nous fait découvrir, avec sa lucidité habituelle, les secrets d'une prodigieuse

(*) L'auteur est un des Régisseurs de la Régie des rentes du Québec. Il a été sous-ministre du Revenu de la province, président national de l'Institut d'Admini:tration publique du Canada, membre du Conseil d'administration et trésorier de l'Institut des comptables agréés et membre du Conseil d'administration de la National Tax Association des Etats-Unis.

(1) John Kenneth Galbraith, The New Industrial State, Houghton Mifflin Company, Boston, 1967, 427 pages. 
croissance qui, en moins d'un siècle, a donné au monde un colosse industriel qui fait tout à la fois sa crainte et son admiration. Orgueilleux bien sûr de nous le faire mieux connaître, il ne peut cependant cacher une certaine inquiétude quant aux dangers que présente pour la société américaine une pieuvre dont les tentacules rejoignent tous les domaines et qui, insidieusement, la domine un peu plus chaque jour. Voyons ce qu'il nous dit.

\section{LA CORPORATION}

Au centre du plus fantastique empire industriel que le monde ait jamais connu, Galbraith place la corporation et il nous fait voir comment, ayant dépassé le stade d'entreprise opérant en vase clos sous la direction d'actionnaires, et à leur seul profit, elle s'intègre maintenant au grand ensemble «état-industrie». Indépendante des marchés financiers pour la plus grande partie des fonds nécessaires à son expansion, elle pratique l'autofinancement, et l'auteur fait remarquer qu'elle constitue le plus vaste réservoir de l'épargne américaine; ainsi, dit-il, dans la seule année 1965 , les revenus utilisés à cette fin furent de l'ordre de $\$ 83$ milliards, alors que l'épargne globale des particuliers atteignait à peine les $\$ 25$ milliards. On voit aussi qu'en 1962, 5 des plus puissantes corporations aux Etats-Unis contrôlaient à elles seules environ $12 \%$ de tous les actifs employés par l'industrie et qu'en portant ce total à 50 et à 500 , les pourcentages devenaient $331 / 3$ et $662 / 3$ respectivement.

Pour être efficiente, nous dit l'auteur, une entreprise manufacturière doit avoir assez d'envergure pour être en mesure de faire face aux dépenses considérables qu'entraîne la constante évolution de la technologie, sans parler des frais de publicité et autres déboursés que nécessite la mise en marché de ses produits. Et, à titre d'exemple, il signale que si en 1903, Ford pouvait opérer avec un capital de $\$ 150,000$. et un personnel de 125 employés, il a fallu, en 1964, plus de $\$ 60$ millions pour lancer sur le marché la * Mustang " (la compagnie avait alors 317,000 ouvriers à son service et un actif global de l'ordre de $\$ 6$ milliards).

Cette nouvelle corporation que nous décrit Galbraith ne compte pas exclusivement sur le management pour toutes ses décisions importantes, mais bien plus sur tout un groupe de technocrates qui oeuvrent à l'intérieur de comités internes qu'il englobe sous le qualificatif de * technostructure »; suivant lui, l'ère du tout-puissant chef d'entreprise, et de l'actionnaire-patron est définitivement chose du passé Et voici ce qu'il dit à son sujet :

* He is a passive and functionless figure, remarkable only in his capacity to share, without effort or even without appreciable risk, in the gains from the growth by which the technostructure measures its success. No grant of feudal privilege has ever equaled, for effortless return, that of the grandparent who bought and endowed his descendants with a thousand shares of General Motors or General Electric. The beneficiaries of this foresight have become and remain rich by no exercise of effort or intelligence beyond the decision to do nothing, embracing as it did the decision not to sell. " (p. 394) 


\section{LE SOCTAYISME}

Quant au socialisme, que trop souvent nous confondons avec nationalisation, l'auteur croit que la formule démocratique américaine est infiniment supérieure à toute autre, car on a su laisser où il se doit le centre des décisions, c'est-à-dire au niveau de la *technostructure * plutôt que d'essayer d'imposer du centre une planification qui, trop souvent, s'est avérée boiteuse; et il ajoute : the genius of the industrial system lies in its organized use of capital and technology (p. 354). Si l'on considère les résultats obtenus, il serait difficile de le contredire sur ce point.

A part cette distinction, sans doute fondamentale, Galbraith ne voit pas très bien en quoi peut différer, dans le contexte économique actuel, la gestion d'une entreprise, qu'elle soit nationalisée ou non. Dans les deux cas, elle dépend sur l'Etat pour :

- assurer l'équilibre entre la demande et la production;

- adopter des mesures visant à stabiliser les prix et les salaires;

- la formation du personnel qualifié dont elle a besoin; et

- l'achat d'une partie importante de la production, à titre bien souvent de principal client.

\section{LA GUERRE ET LA DÉREENSE}

Concernant les dépenses encourues pour la guerre et la défense, l'argumentation de Galbraith, si réaliste soit-elle, n'est pas sans nous laisser songeur...

Sans nier qu'il soit possible et même souhaitable de voir se comprimer les dépenses de cette nature, comme il l'a d'ailleurs fait à plusieurs reprises concernant la guerre au Vietnam, il laisse entendre qu'il serait très difficile, sinon impossible, de canaliser des sommes qui absorbent présentement plus de $50 \%$ du budget vers des secteurs qui avantageraient aussi directement l'avancement de la technologie américaine; ce n'est sûrement pas, dit-il, en détournant ces fonds vers l'habitation, la sécurité sociale ou les loisirs, que l'on atteindrait les mêmes objectifs ...

On peut se demander si nous n'avons pas là une réponse, du moins partielle, à cette interminable guerre du Vietnam, et à la course aux armements que le monde a connue depuis le dernier conflit mondial. Voilà de quoi nous faire réfléchir...

\section{LE SYNDICALISMME}

Galbraith croit que le mouvement syndical est en perte de vitesse dû à l'avancement de la technologie moderne (cybernétique, automation, 
etc.), le haut niveau de l'embauchage et la stabilité des marchés qui assure à l'industrie une économie grandement dirigée. Et voici d'ailleurs, quelques statistiques qu'il porte à notre attention :

- de 1956 à 1962, le nombre des ouvriers (blue collars) est passé de 16.6 à 14.9 millions, soit une baisse de 1.7 million, alors que l'ensemble de la main-d'oeuvre (excluant les exploitations agricoles) accusait une augmentation dépassant 4 millions;

- seulement $12 \%$ des * collets blancs * sont syndiqués et, pour l'industrie prise séparément, ce pourcentage n'est que de $5 \%$;

- de 1947 à 1965, le nombre " collets blancs " professionnels, administrateurs, employés de bureau, vendeurs, etc. augmenta de 9.6 millions alors que les " collets bleus » accusèrent une baisse de 4 millions; et en 1965, ce nombre excédait de 8 millions ceux de toutes les autres catégories.

Cette marche arrière serait selon lui irréversible - * The loss of union membership is not a temporary setback pending the organization of white-collar employees and engineers but the earlier stages of a permanent decline... (p. 263); et il ajoute — - The union is much less essential for the worker. What the technostructure gives to the union, it can also give without a union or to avoid having a union. At a minimum the union shrinks in stature ... (pp. 265-266)

Mais il a quand même de bonnes choses à dire concernant ce que nous devons aux syndicats, par exemple _ $\ldots$... they have helped to arrange a trade of higher pay, a shorter week, severance pay or other provision for those sacrificed for smaller employment. And they have persuaded their members to accept the bargain. The industrial system attaches great importance to such help. The union leader who provides it is accorded its highest encomium, that of labor statesman ». (p. 277)

\section{L'ENSEIGNEMENT}

$\mathrm{Au}$ domaine de l'enseignement, Galbraith voit dans l'orientation du système éducatif, la seule planche de salut qui empêchera ses concitoyens de devenir tout simplement les roues d'engrenage d'un puissant empire économique. - - Its requirements are twofold. The first is comprehension and skepticism which insure that there will be systematic questioning of the beliefs impressed by the industrial system. The second is a political pluralism which voices the ideas and goals of those who, industrially speaking, choose to contract out of the industrial system ». (p. 370)

Il s'inquiète de voir nos centres d'éducation supérieure axer la très grande partie de leurs efforts sur l'économique et reléguer au second plan les arts et les humanités, et voit s'amorcer une violente réaction contre une orientation qui est en opposition directe aux profondes aspi- 
rations de l'homme. - $<$ He (the educator) must realize this, and exert his power not on behalf of the industrial system, but on behalf of the entire human personality $\gg$. (p. 372)

Le progrès économique, si important dit-il, est loin d'être le seul critère d'une civilisation avancée; et il ajoute avec humour - . This is the modern morality. St. Peter is assumed to ask applicants only what they have done to increase the G.N.P. > (p. 408)

Galbraith voudrait que les universités soient complètement libres de toute attache, tant sur le plan financier quautrement, sans quoi dit-il - a the university will become a shell with which they have only a residential association *. (p. 373) Et il ajoute - * the growth and influence of college and university communities are in response to the needs of the industrial system. But this does not necessarily create a primary obligation to the needs of the industrial system. Gratitude and death do not exist as between social institutions. The only reality is the right social purpose ... (p. 376)

\section{L'ÉmANGäatton}

Galbraith, on le sent, craint le conflit qui s'annonce entre les tenants de la domination de la société par l'économique et ceux qui veulent y trouver un horizon plus vaste. Esclave ou maître. Voilà la question qui se pose à une grande partie de la population, surtout aux jeunes qui n'acceptent pas de se voir sacrifiés aux appétits de notre moloch moderne. A moins d'un virage voulu et nécessaire, une violente réaction pointe à l'horizon, et l'impérialisme économique américain pourrait bien porter en lui les germes de sa propre destruction...

Une prise de conscience s'impose, et Galbraith résume ainsi sa pensée : "If economic goals are the only goals of society it is natural that the industrial system should dominate the state and the state should serve its ends. If other goals are strongly asserted, the industrial system will fall into its place as a detached and autonomous arm of the state, but responsive to the larger purposes of society. We have seen wherein the chance for salvation lies. The industrial system, in contrast with its economic antecedents, is intellectually demanding. It brings into existence, to serve its intellectual and scientific needs, the community, that, hopefully, will reject its monopoly of social purpose .. (p. 399)

Galbraith est un de ces spécialistes dont le sens de l'observation s'étend au-delà de sa spécialité (chose plutôt rare de nos jours) et, un peu comme notre propre Stephen Leacock, il voit l'économique dans le cadre d'un grand tout à la dimension globale de l'homme qui, sans cesse, recherche son mieux être. J'ouvrirais ici une parenthèse pour dire que je conçois très bien qu'un économiste soit à ses heures philosophe, sociologue, historien et même humoriste, cela n'est pas nécessai- 
rement contradictoire, et c'est en étant un peu tout cela qu'il pourra peut-être mieux comprendre et mieux servir notre grande farnille humaine...

Dans ce livre que doivent lire tous ceux que le phénomène américain intéresse Galbraith signale la complète domination par le complexe industriel de ce qu'il appelle « The New Industrial State ». Et il insiste sur le fait que même en laissant de côté les nationalisations dont il ne voit pas les avantages, il reste quand même vrai que le grand ensemble économique des U.S.A. ne saurait donner son plein rendement, dans le contexte actuel, sans l'étroite collaboration de cet important partenaire qu'est devenu l'Etat. Cependant il ne fait aucun doute suivant lui que la grande réussite du système économique de nos voisins est d'avoir su conserver à une entreprise privée restructurée, cette liberté de décision qui lui vaut un dynamisme et un leadership, jusqu’à date, incontesté. Ses réflexions à ce sujet devraient faire réfléchir ceux qui sont convaincus d'avoir trouvé dans la nationalisation une recette passe-partout.

Galbraith voit dans la recherche une autre raison de la prédominance économique de son pays (il est intéressant de signaler qu'en 1962 on y consacrait près de $\$ 94$ par tête à comparer à environ $\$ 15$ au Canada; cela représentait en $19592.5 \%$ de leur produit national brut en regard de $0.72 \%$ chez nous). Nous avons là une explication de la prépondérance de ce * know how "américain qui fait notre envie et sans l'aide duquel notre développement économique accuserait un retard considérable.

On aurait aimé que l'auteur précise davantage sa pensée sur certains des problèmes qu'il soulève : l'indépendance financière des universités; les droits de l'actionnaire moderne; le plein emploi et l'inflation; les monopoles déguisés, et surtout lorsqu'il nous parle de la guerre et de sa dimension économique, car il sera toujours difficile à un chrétien sincère d'accepter comme normal la terrible chevauchée de ce cavalier apocalyptique. Espérons que cela nous vaudra un nouveau livre.

Servan Schriher a raison de sonner l'alarme dans son dernier ouvrage Le Défi Américain qui, sentons-nous, a été pour partie inspiré de l'étude de Galbraith. Mais s'il faut, comme il le laisse entendre, combattre le colosse américain en utilisant les formules qui lui ont permis d'atteindre sa stature, sachons tout de même comprendre le message de Galbraith, et visons à atteindre nos objectifs en préservant ces libertés fondamentales sans lesquelles il est impossible à l'homme d'atteindre son plein épanouissement. 